\title{
UTILIDAD DEL TOMÓGRAFO DE COHERENCIA ÓPTICA (OCT) EN EL SEGUIMIENTO DE LA HIPERTENSIÓN INTRACRANEAL IDIOPÁTICA EN LA INFANCIA
}

\section{UTILITY OF OPTIC COHERENCE TOMOGRAPHY (OCT) IN THE FOLLOW-UP OF IDIOPATHIC INTRACRANIAL HYPERTENSION IN CHILDHOOD}

\author{
SÁNCHEZ-TOCINO H ${ }^{1}$, BRINGAS R ${ }^{2}$, IGLESIAS D ${ }^{1}$, GONZÁLEZ-PÉREZ A ${ }^{3}$, \\ DEL VILLAR-GALÁN R ${ }^{3}$
}

\begin{abstract}
RESUMEN
Objetivo: Mostrar la utilidad de la tomografía de coherencia óptica (OCT) en el seguimiento de la hipertensión intracraneal idiopática (HII) en la edad pediátrica.

Métodos: Se ha realizado el diagnóstico y posterior seguimiento, del episodio agudo de HII en tres niñas con edades entre 4 y 11 años. Todas acudieron con síntomas inespecíficos: Alteraciones visuales, cefalea, abdominalgia, visión doble y tortícolis. Los estudios de neuroimagen fueron normales. Tras la punción lumbar, la presión intracraneal estaba elevada. Se realizó campo visual (CV) mediante perímetro Octopus y medida de la capa de fibras nerviosas (CFN) con OCT en todas las revisiones.

Resultados: La agudeza visual fue normal en todas ellas, con paresia de VI par en dos casos. El estudio de fondo de ojo reveló siempre un gran edema de papila. La medida inicial de la CFN con OCT se halló elevada, duplicando o triplicando los valores normales. El seguimiento y tratamiento con predni-
\end{abstract}

\begin{abstract}
Purpose: To show the utility of optic coherence tomography (OCT) in the follow up of the idiopathic intracranial hypertension (IIH) in childhood. Methods: Three girls, aged between 4 and 11 years, were diagnosed and subsequently followed with the help of OCT for an episode of idiopathic intracranial hypertension. All of them had presented with non-specific symptoms such as visual disturbance, headache, stomach ache, and in one case diplopia and torticolis. Brain imaging studies were normal, however lumbar puncture found an elevated intracranial pressure. Visual fields were tested using Octopus automated perimetry and the optic nerve fiber layer (ONF) was measured by OCT at each of the attendances.

Results: The visual acuity was normal in all cases, but two girls had evidence of an abducens nerve palsy. Fundus biomicroscopy revealed marked papilledema. The thickness of the ONF was increased 2-3 times over normally expected levels. The
\end{abstract}

\footnotetext{
Recibido: 16/12/05. Aceptado: 17/7/06.

Departamentos de Oftalmología y Pediatría del Hospital Río Hortega. Valladolid. España.

1 Doctor en Medicina. Especialista en Oftalmología.

2 Licenciado en Medicina. Especialista en Oftalmología

3 Licenciado en Medicina. Especialista en Pediatría.

Comunicación presentada en el LXXXI Congreso de la S.E.O. (Zaragoza 2005).

Correspondencia:

Hortensia Sánchez Tocino

Servicio de Oftalmología. Hospital Río Hortega

Avda. Cardenal Torquemada, s/n

47010 Valladolid

España

E-mail: hsanchez@auna.es
} 
sona y acetazolamida vía oral se realizó de acuerdo a las medidas del OCT. La evolución fue buena con progresiva normalización de los valores de grosor en el OCT. Si bien en un caso, tras 3 meses sin tratamiento, se detectó mediante OCT una recurrencia asintomática.

Conclusiones: La HII en edad pediátrica es una entidad poco frecuente y diferente de la afectación típica del adolescente y el adulto. El OCT es una técnica objetiva y reproducible que puede ayudar a completar el diagnóstico y, sobre todo, el posterior seguimiento clínico y terapéutico de estos cuadros en niños.

Palabras clave: Tomografía de coherencia óptica en niños, hipertensión intracraneal idiopática, neuropatía en niños, papiledema, hipertensión intracranial benigna en la infancia, pseudotumor cerebri. follow up and the treatment with prednisone and azetazolamide was monitored by OCT, with a good outcome and return of the ONF levels to normal. In one case, however, a recurrence was detected after three months without treatment.

Conclusion: IIH in childhood is an uncommon condition, often with a different presentation from what is seen in teenagers and adults. OCT is a useful technique helping in both the diagnosis and the follow-up of this disorder in children (Arch Soc Esp Oftalmol 2006; 81: 383-390).

Key words: Optic coherence tomography in children, idiopathic intracranial hypertension, neuropathy in a child, papilledema, benign childhood intracranial hypertension, pseudotumor cerebri.

\section{INTRODUCCIÓN}

Se define la hipertensión intracraneal idiopática (HII), o pseudotumor cerebri como una elevación anormal de la presión intracraneal, no asociada a un proceso expansivo o a una hidrocefalia, con un liquido cefalorraquídeo de composición normal $(1,2)$. Se presenta como un edema de papila asociado o no a pequeñas hemorragias peripapilares, sin defecto pupilar aferente y con poca afectación de la agudeza visual, al menos inicialmente.

Se muestra, a propósito de tres casos, la utilidad de la tomografía de coherencia óptica (OCT) como complemento en el diagnóstico y, sobre todo, en el seguimiento de esta patología en la edad pediátrica. Con OCT puede observarse un aumento muy importante del grosor de la capa de fibras nerviosas (CFN), muy superior a la encontrada en los cuadros de neuritis anteriores.

\section{SUJETOS, MATERIAL Y MÉTODOS}

Se describen tres casos de mujeres, con edades comprendidas entre 4 y 11 años, que acudieron a la consulta de urgencias por síntomas inespecíficos visuales y sistémicos. Se realizó, en todos los casos, un estudio oftalmológico completo con toma de agudeza visual (AV) mediante test de Snellen, estudio de los movimientos oculares, cover test, test de diplopía de lejos con luces de worth, biomicroscopía anterior, fondo de ojo con lente de $90 \mathrm{Dp}$, campo visual $(\mathrm{CV})$ mediante perímetro automático Octopus (OCTOPUS, Haag-Streit AG, Koeniz-Berne, Switzerland) y estudio de CFN con OCT.

Se realizó la medición del grosor de la capa de fibras nerviosas de la retina mediante tomógrafo óptico de coherencia (OCT-3, Carl Zeiss, Meditec, Dublín, CA, USA). La OCT es un método de diagnóstico por imagen no invasivo que realiza medidas cuantitativas objetivas y directas de la papila y de la CFN. Se basa en un principio óptico denominado interferometría de baja coherencia, utilizando un haz de luz de $820 \mathrm{~nm}$ de longitud de onda. Una parte del haz de luz se dirige hasta la retina y otro se dirige hacia un espejo de referencia, que se sitúa a una distancia conocida. Los ecos obtenidos tras reflejarse en la retina se comparan con los provenientes del espejo de referencia produciéndose un fenómeno de interferencia que es captado y medido por un detector. Así, se obtienen imágenes bidimensionales compatibles con imágenes histológicas con un corte axial de unas $10 \mu \mathrm{m}$ aproximadamente y una profundidad de $2 \mathrm{~mm}$ mediante barridos que oscilan entre 128 y $768 \mathrm{~nm}$ (3). Aunque es ideal realizar la prueba en condiciones de midriasis, pueden obtenerse buenas imágenes tomográficas sin necesidad de dilatar la pupila. Para el estudio de la estructura de la CFN, el OCT detecta la superficie anterior de la CFN y el epitelio pigmentario de la retina (EPR). 
Estas estructuras, al tener una mayor reflectividad, aparecen dibujadas en color rojo. En el estudio de la CFN los parámetros de grosor parapapilar son automáticamente calculados y evaluados en el eje vertical y horizontal a lo largo de un scan circular. Se utilizó para realizar las mediciones, el modo «Fast RNFL Thickness (3.4)» bajo midriasis obtenida con colirio de ciclopentolato 1\% (Colircusi ciclopléjico 1\% Alcon Cusí, El Masnou, Barcelona, España). El análisis de las mediciones se realizó mediante el protocolo «RFNL Thickness Average Análisis» que puede cuantificar el grosor promedio en micras $(\mu)$ total, en 4 sectores peripapilares: inferior, superior, nasal y temporal y en nueve husos horarios; y el «RNFL Thickness Serial Analysis Report» para el estudio de las diferencias en el grosor en las distintas exploraciones realizadas.

El servicio de pediatría de nuestro hospital realizó, en todos los casos, una analítica general que incluyó: hemograma, proteína $\mathrm{C}$ reactiva, factor reumatoide, proteinograma, ANA, antiDNA, hormonas tiroideas, cortisol, ASLO, serología a virus herpes, zoster, citomegalovirus, toxoplasmosis, legionella, coxiella, chlamydia, mycoplasma, borrelia, sífilis, brucella, salmonella, rickerttsia e hidatidosis; estudios de imagen: tomografía axial computerizada (TAC), resonancia nuclear magnética (RNM) y angioresonancia; pruebas electrofisiológicas: Electroencefalograma; y una punción lumbar, valorando la presencia de proteínas, glucosa, células y la medida de la presión intracraneal. En los tres casos todos los estudios resultaron normales excepto la cifra de presión intracraneal que resultó elevada. La presión intracraneal se tomó en el espacio subaracnoideo mediante un sistema hidráulico (columna llena de líquido) en $\mathrm{cm}$ de agua.

\section{RESULTADOS}

\section{Caso n. ${ }^{\circ} 1$.}

Niña de 10 años de edad que acudió por abdominalgia. A la exploración oftalmológica: AV de la unidad en ambos ojos (AO), leve paresia del VI par craneal que causaba diplopía en posiciones extre-

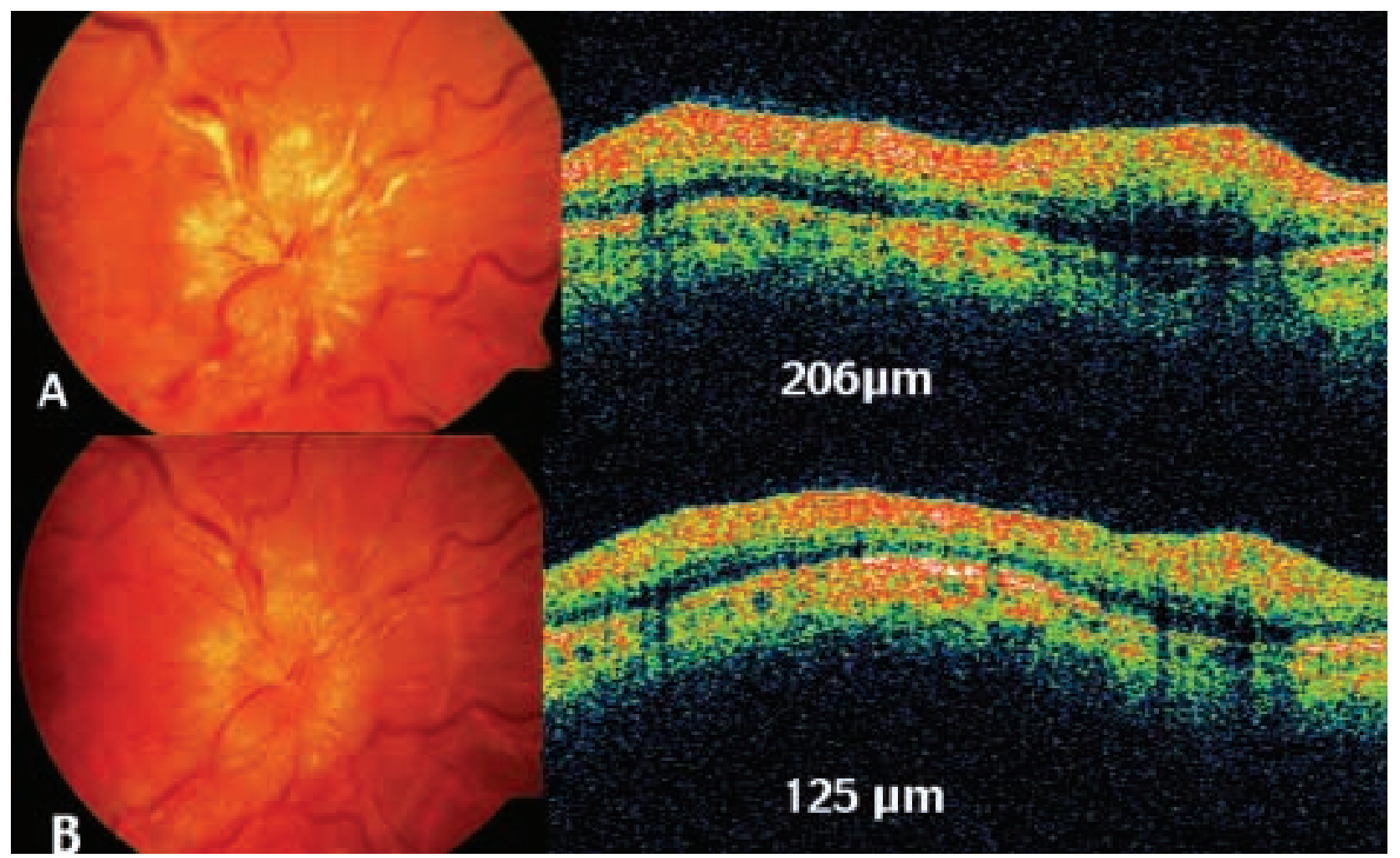

Fig. 1: Caso número 1: A. Edema de papila bilateral e imagen de OCT con engrosamiento de CFN inicial en OD. B. Papila e imagen de OCT al mes en OD. Normalización de CFN tras el tratamiento. 
mas. En el fondo de ojo se evidenció un edema de papila bilateral (fig. 1). El CV mostró un aumento de la mancha ciega. La OCT evidenció un engrosamiento difuso de la CFN que mantiene el patrón típico de doble joroba, grosor medio de la CFN 206 $\mu \mathrm{m}$ y $108 \mu \mathrm{m}$ ojo derecho (OD) e izquierdo (OI) respectivamente (fig. 1). Las técnicas de neuroimagen, la analítica y el estudio bioquímico del liquido cefalorraquídeo (LCR) fueron normales. La presión intracraneal fue de $31 \mathrm{~cm}$ de H20. Indice de masa corporal (IMC) 17,6 (percentil 50-75).

\section{Caso n. ${ }^{\circ} 2$}

Niña de 4 años de edad que fue atendida por diplopía, tortícolis y miodesopsias. A la exploración oftalmológica la AV fue de unidad en AO. Paresia del VI par de ojo izquierdo, tortícolis con cara lado derecho. Test luces de Worth patológico. En el estudio del fondo de ojo se objetivo edema de papila bilateral con una pequeña hemorragia en astilla en el polo inferior de la papila del ojo izquierdo. Dada la corta edad de esta niña los campos visuales realizados no se pudieron considerar como fiables. La OCT reveló un gran aumento de la CFN, media de grosor de la capa de fibras del nervio óptico $200 \mu \mathrm{m}$ y $245 \mu \mathrm{m}$ en OD y OI respectivamente (fig. 2). El LCR, el estudio analítico y de neuroimagen fueron normales. La presión intracraneal fue de $45 \mathrm{~cm}$ de H20. IMC: 18,6 (percentil 9095).

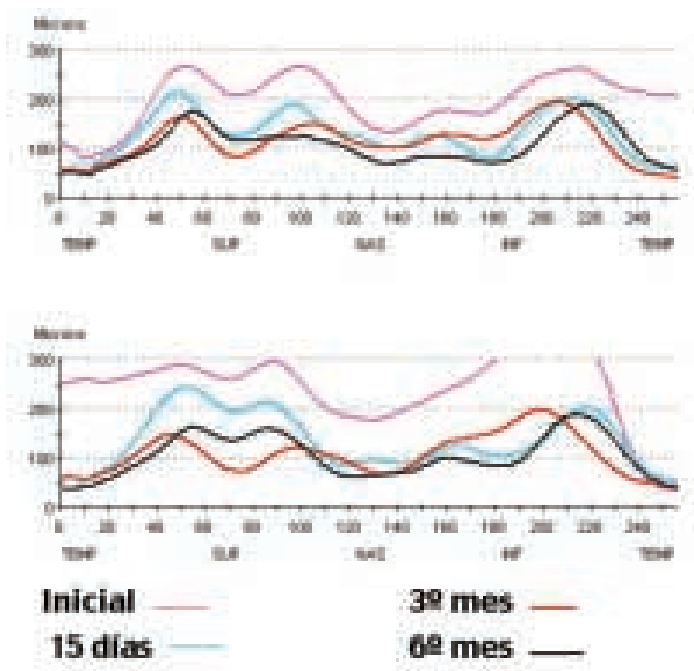

Fig. 2: Caso número 2: Evolución de la CFN: Inicial, 2 semanas, $3 .^{\circ}$ mes y $6 .^{\circ}$ mes.

\section{Caso n. ${ }^{\circ} 3$}

Paciente de 11 años de edad, diagnosticada previamente de retinitis pigmentosa, que acudió a una consulta de revisión refiriendo visión borrosa, mareo y episodios de amaurosis fugax en los últimos días.

A la exploración presentó una $\mathrm{AV}$ de 0,7 en AO, sin cambios con respecto a controles previos. Sin alteraciones en los movimientos oculares ni diplopia. El fondo de ojo mostró un edema de papila bilateral, dispersión de pigmento de tipo espicular retroecuatorial en los cuatro cuadrantes y máculas de aspecto normal (fig. 3). El CV presentó una restricción periférica propia de la retinitis pigmentosa. La OCT evidenció un gran aumento en el grosor medio de la CFN de $546 \mu \mathrm{m}$ en el OD y $442 \mu \mathrm{m}$ en el OI (fig. 3). El estudio analítico y de neuroimagen fueron normales. La punción lumbar mostró una bioquímica normal y una presión de $30 \mathrm{~cm}$ de $\mathrm{H} 20$. IMC: 29,19, sobrepeso (percentil 97).

En todos los casos se instauró un tratamiento, por vía oral, con prednisona a una dosis de 1,5 $\mathrm{mg} / \mathrm{kg} / \mathrm{d}$ ía, acetazolamida $250 \mathrm{mg} / 8$ horas, suplemento de potasio y protección gástrica por parte del Servicio de Pediatría de nuestro Hospital. El edema de papila y la paresia del VI par mejoraron, en los dos primeros casos, pasada la primera semana. Se realizaron controles con OCT a la semana, a los 15 días, al mes y, posteriormente, de forma mensual. La figura 2 muestra la evolución del OCT en el caso número 2, se observa una progresiva disminución del grosor de la CFN. En el caso número 3 el edema de la papila se mantuvo durante ocho semanas. El seguimiento máximo fue de 9 meses (fig. 3). Se comenzaron a retirar los corticoides, en pauta descendente, cuando la clínica mejoró y se evidenció una disminución significativa del grosor de la CFN en el OCT y la acetazolamida cuando se comprobaron medidas de CFN en el OCT en torno a $110 \mu \mathrm{m}$. En los dos primeros casos esto se consiguió en torno al tercer mes, en el caso número 3 el tratamiento se prolongó 6 meses.

En el caso número 1, tras 3 meses sin tratamiento, se detectó mediante OCT un pequeño aumento transitorio de la CFN que, al no producir sintomatología, no requirió tratamiento. Sin embargo, en el caso número 3, a los dos meses de concluido el tratamiento se ha observado una disminución en el espesor de la CFN hasta cifras de $75 \mu \mathrm{m}$ en el ojo derecho y $67 \mu \mathrm{m}$. en el ojo izquierdo (fig. 4), que 


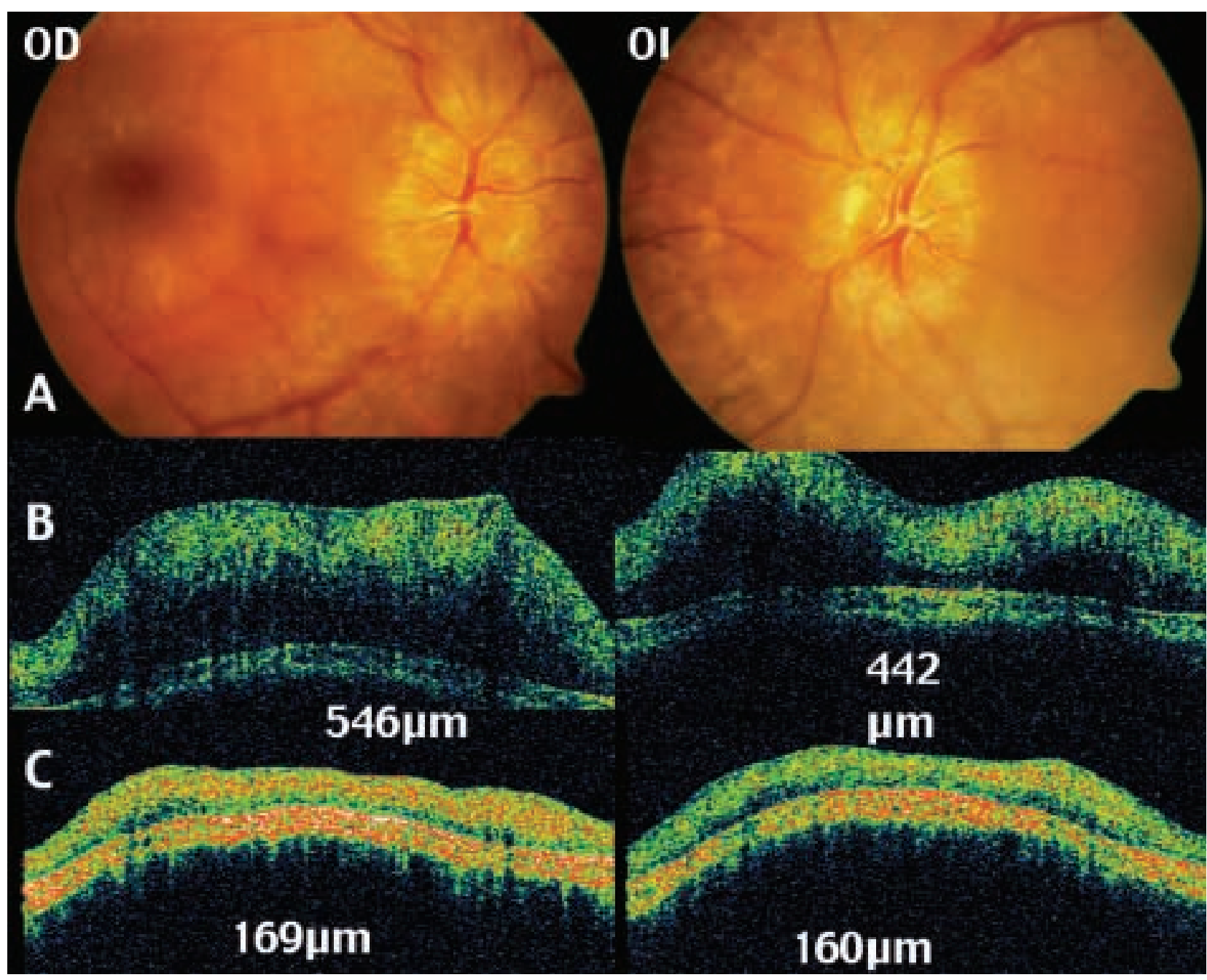

Fig. 3: Caso número 3: A. Edema de papila bilateral. B. Imagen de OCT con engrosamiento inicial de CFN en A.O. C. OCT con $C F N$ al $6^{\circ}$ mes en A.O.

reflejan la evolución a un cierto grado de atrofia. En el seguimiento a más largo plazo 1 año ni en el caso 1 ni en el 2 se ha detectado mediante OCT ninguna recurrencia importante que requiriera de nuevo tratamiento.

\section{DISCUSIÓN}

La HII es una entidad que cursa con elevación de la presión intracraneal sin que exista una causa aparente y con estudio de imagen normal del sistema nervioso central. Esta enfermedad está bien descrita en adultos donde existe, de forma característica, una predominancia femenina y una asociación con la obesidad. En la edad pediátrica las características pueden diferir. Presenta una igual distribución por sexo; en la serie publicada por Orssaud describe que el $47 \%$ de los 30 pacientes estudiados eran mujeres menores de 13 años mientras que el $75 \%$ de los mayores de 13 años eran mujeres $(4,5)$. En adolescentes, al igual que en los adultos, esta enfermedad se relaciona con la obesidad y tiene una preponderancia femenina.

En niños prepuberales la HII generalmente se presenta asociada a cefalea, diplopia, estrabismo y en algunos casos puede existir rigidez de nuca. Se han descrito también otras manifestaciones generales como irritación, agresividad, alteraciones del comportamiento o somnolencia (6).

Baker ha publicado una perdida de agudeza visual entre el 13 y el 38\% de los niños con HII (7). 


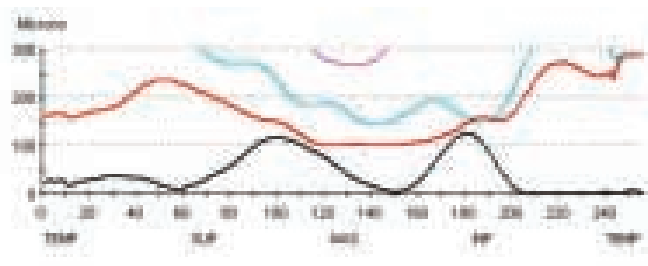

OD

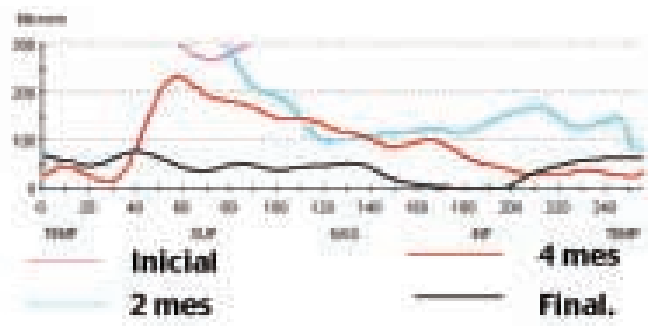

os

Fig. 4: Caso número 3. Evolución de la CFN en los 9 meses.

Cinciripini encontró defectos en el campo visual en el $85 \%$ de los ojos, siendo el defecto más frecuente un aumento de la mancha ciega (6). No se encontró una perdida de $\mathrm{AV}$ en ningún caso de los aquí presentados. Existió un defecto inicial del CV en el caso número 1 que se recuperó totalmente. En el caso número 3, la afectación campimétrica estaba asociada a la retinitis pigmentosa ya conocida. No se detectaron ni con el estudio de tomografía ni por OCT la presencia de drusas del nervio óptico.

Si bien en la mayoría de las series publicadas se describe una recuperación completa de la agudeza visual, del estrabismo o de los defectos del CV, existen casos con pérdidas permanentes de AV (4) o del campo (6), siendo el diagnóstico y el manejo precoz muy importante en la resolución completa del cuadro. En nuestra serie, solo en el caso número 3 se ha detectado una perdida de CFN permanente, que podríamos determinar como secuela del cuadro agudo de HII, sin modificación de la AV. Sin embargo, con la patología previa de la paciente y sin el OCT previo, no se pudo negar la posibilidad de que la cifra obtenida al realizar la medición de la CFN pueda ser la que existía de base en este caso. Ya otros autores han descrito la imposibilidad de conocer mediante OCT si la reducción del grosor de fibras del nervio óptico seguido por OCT se debe a una disminución del edema o una atrofia de las mismas (8). Sin embargo, cuando el OCT se correlaciona con otros hallazgos tales como campo visual, puede ser bastante útil para el seguimiento de pacientes con papiledema (8).
La normalización de la papila ocurre en, aproximadamente, 4 meses si el tratamiento tuvo una instauración rápida. Pero la evolución, en algunos casos, puede ser hacia el desarrollo de una atrofia del nervio óptico (6). Sin embargo, no existe una correlación entre la importancia del edema de papila y la elevación de la presión o entre este y el riesgo de alteración de la función visual. Por otra parte las alteraciones del CV tampoco son específicas del cuadro (4).

Si bien es cierto que el OCT no permite diferenciar claramente un papiledema sobre todo si es leve de un seudopapiledema, elevación congénita del nervio óptico, como ya ha sido demostrado por otros autores (9), ya que únicamente nos detecta que existe un aumento en el grosor de la capa de fibras del nervio óptico. Tampoco en estos casos en los que detectemos en el fondo de ojo un edema de papila que se acompaña de un cuadro clínico más o menos manifiesto de cefalea con o sin disminución de visión, el OCT va evitar la realización de pruebas de neuroimagen; sin embargo, si nos va a permitir de forma sencilla poder hacer el seguimiento hasta llegar a la estabilización del cuadro (8). Karma et al. (9) también señalan que es probable que el grosor de fibras nerviosas en pacientes con papiledema más severo pueda ser mayor con diferencias significativas estadísticamente respecto de los ojos con seudopapiledema.

Se muestra en esta breve revisión que el OCT representa una prueba muy útil en la HII, ya que es una técnica objetiva, no invasiva y reproducible, que se puede realizar en niños y que permite apoyar el diagnóstico ante síntomas inespecíficos y, sobre todo, ha demostrado su gran utilidad en el seguimiento y valoración de la eficacia terapéutica sobre el edema de papila, reflejada por una normalización de la CFN. Asimismo, nos ha permitido la detección de recurrencias y el descubrimiento de secuelas crónicas tras un episodio agudo de HII.

\section{BIBLIOGRAFÍA}

1. Biousse V, Bousser MG. L'hypertension intracrânienne bénigne. Rev Neurol (Paris) 2001; 157: 21-34.

2. Wall M, George D. Idiopathic intracranial hypertension. A prospective study of 50 patients. Brain 1991; 114: 155180.

3. Chauhan DS, Marshall J. The interpretation of optical coherence tomography images of the retina. Invest Ophthalmol Vis Sci 1999; 40: 2332-2342. 
4. Orssaud C, Dureau P, Zerah M, Cinalli G, Sainte Rose C, Kahn AP, et al. Benign childhood intracranial hypertension. J Fr Ophtalmol 2001; 24: 54-59.

5. Wall M. Idiopathic intracranial hypertension. Neurol Clin 1991; 9: 73-95.

6. Cinciripini GS, Donahue S, Borchert MS. Idiopathic intracranial hypertension in prepubertal pediatric patients: characteristics, treatment, and outcome. Am J Ophthalmol 1999; 127: 178-182.

7. Baker RS, Carter D, Hendrick EB, Buncic JR. Visual loss in pseudotumor cerebri of chidhood. A follow-up study. Arch Ophthalmol 1985; 103: 1681-1686.

8. Hedges TR. Optical coherence tomography in neuroophthalmology. In: Schuman JS, Puliafito CA, Fujimoto JG. Optical Coherence tomography of ocular diseases. 2nd ed. Thorofare: SLACK Incorporated; 2004; 13: 611630.

9. Karam EZ, Hedges TR. Optical coherence tomography of the retinal nerve fibre layer in mild papilloedema and pseudopapilloedema. Br J Ophthalmol 2005; 89: 294-298. 